\title{
OUTERPLANAR COARSENESS OF PLANAR GRAPHS
}

\author{
PAUL C. KAINEN
}

\begin{abstract}
The (outer) planar coarseness of a graph is the largest number of pairwise-edge-disjoint non-(outer)planar subgraphs. It is shown that the maximum outerplanar coarseness, over all $n$-vertex planar graphs, lies in the interval $[\lfloor(n-2) / 3\rfloor,\lfloor(n-2) / 2\rfloor]$.
\end{abstract}

\section{INTRODUCTION}

A graph $H$ is outerplanar if the graph $H * K_{1}$, consisting of the join of $H$ with an isolated vertex, is planar. Some invariants related to outerplanarity are bounded on the family of all planar graphs; e.g., Yannakakis [6] showed that the book thickness of a planar graph is at most 4, and the famous CGH-conjecture [1], that every planar graph has outerplanar thickness at most 2, may have at last been proven by Goncalves [2]

However, the worst-case outerplanar crossing number grows quadratically with the number of vertices of the planar graph. Indeed, as the number of edges in a planar graph is less than 3 times the number $n$ of vertices, the outerplanar crossing number is less than $(9 / 2) n^{2}$, and we showed in [5] that the family $G_{n}$ of $n$-vertex planar graphs has outerplanar crossing number $c r_{o p}\left(G_{n}\right)$ asymptotically equal to $n^{2} / 4$, where $G_{n}$ is the join of two isolated vertices with an (n-2)-cycle, i.e., $G_{n}=C_{n-2} * \bar{K}_{2}$. In fact, [5] gives an exact formula for $n \geq 5$,

$$
c r_{o p}\left(G_{n}\right)=\left\lfloor\frac{n-2}{2}\right\rfloor\left\lfloor\frac{n-3}{2}\right\rfloor+2 n-8 .
$$

We show that worst-case outerplanar coarseness of planar graphs grows linearly with $n$.

\section{Outerplanar coarseness of $G_{n}$}

The outerplanar coarseness of a graph $G$ is the largest number of pairwiseedge-disjoint non-outerplanar subgraphs of $G$. As $G$ is outerplanar if and only if it has no subgraph homeomorphic to $K_{4}$ or $K_{2,3}$ [4, p. 107], it follows (Guy [3]) that $G$ with $m$ edges has outerplanar coarseness $\xi_{\text {op }}(G)$ at most $m / 6$. This gives the upper bound for $n$-vertex planar graphs asserted in the abstract. The following theorem provides the lower bound. 


\section{P. C. KAINEN}

Theorem 2.1. Let $n \geq 5$. Then $\xi_{o p}\left(G_{n}\right)=\lfloor(n-2) / 3\rfloor$.

Proof. The $\geq$ inequality is obvious: Take any family of the form $\left\{\bar{K}_{2} *\right.$ $W: W \in \mathcal{W}\}$, where $\mathcal{W}$ is a maximal collection of pairwise-vertex-disjoint 3-element subsets of $V\left(C_{n-2}\right)$ and $\bar{K}_{2}$ denotes the same pair of vertices given in the definition of $G_{n}$. For the reverse inequality, note that a $K_{4^{-}}$ homeomorph in $G_{n}$ includes the entire $n$-2-cycle so $G_{n}$ contains only one such subgraph. Hence, for $n \geq 5$, to maximize the number of pairwise edgedisjoint non-outerplanar subgraphs, one can use homeomorphs of $K_{2,3}$ so $\leq$ holds as well.

We conjecture that $G_{n}$ maximizes $c r_{o p}(G)$ and $\xi_{o p}(G)$ over all $n$-vertex planar graphs $G$.

\section{REFERENCES}

[1] G. Chartrand, D. P. Geller, and S. Hedetniemi, Graphs with forbidden subgraphs, J. Comb. Th. B, 10 (1971), 12-41.

[2] D. Goncalves, Edge partition of planar graphs into two outerplanar graphs, STOC, ACM, 2005, pp. 504-512.

[3] R. K. Guy, Outerthickness and outercoarseness of graphs, Combinatorics, T. P. McDonough and V. Mavron, Eds., Cambridge Univ. Press, 1974, pp. 57-60.

[4] F. Harary, Graph Theory, Addison-Wesley, Reading, MA, 1970.

[5] P. C. Kainen, Outerplanar crossing numbers of planar graphs, Bull. Inst. Combin. Appl., 61 (2011) 69-76.

[6] M. Yannakakis, Embedding planar graphs in four pages, J. of Computer and Syst. Sci., 38 (1989), 36-67.

MSC2010: 05C10

Key words and phrases: Coarseness, crossing number, outerplanar invariants, planar graphs

Department of Mathematics and Statistics, Georgetown University, WashINGTON, D.C. 20057-1233

E-mail address: kainen@georgetown.edu 